\title{
Correction: Cancer from the perspective of stem cells and misappropriated tissue regeneration mechanisms
}

\author{
Mariusz Z. Ratajczak ${ }^{1,2} \cdot$ Kamila Bujko $^{1} \cdot$ Aaron Mack $^{1} \cdot$ Magda Kucia $^{1,2} \cdot$ Janina Ratajczak $^{1}$ \\ Published online: 7 March 2019 \\ (c) The Author(s) 2019
}

Correction to: Leukemia (2018) 32:2519-2526;

https://doi.org/10.1038/s41375-018-0294-7;

published online 30 October 2018
The original version of this Article omitted the following from the Acknowledgements:

OPUS grant UMO-2016/21/B/NZ4/00201 was awarded to MK.

Mariusz Z. Ratajczak

mzrata01@louisville.edu

1 Stem Cell Institute, Division of Hematology and Oncology, James Graham Brown Cancer Center, University Louisville, 500 South Floyd Street, Louisville 40202 Kentucky, USA

2 Department of Regenerative Medicine, Center for Preclinical Research and Technology, Warsaw Medical University, Warsaw, Poland

\section{Correction: Management of relapsed and refractory multiple myeloma: novel agents, antibodies, immunotherapies and beyond}

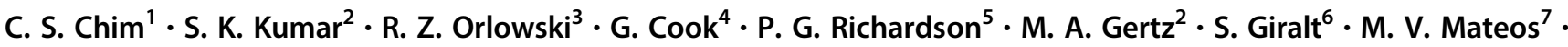 \\ X. Leleu $^{8} \cdot$ K. C. Anderson ${ }^{5}$ \\ Published online: 6 March 2019 \\ (c) The Author(s) 2019. This article is published with open access
}

C. S. Chim

jcschim@hku.hk

1 Department of Medicine, Queen Mary Hospital, The University of Hong Kong, Hong Kong, Hong Kong

2 Department of Medicine, Mayo Clinic at Rochester, Rochester, MN, USA

3 Department of Lymphoma/Myeloma, Division of Cancer Medicine, The University of Texas MD Anderson Cancer Center, Houston, TX, USA

4 Haematology \& Myeloma Studies, Section of Experimental
Haematology, Leeds Institute of Cancer and Pathology, University of Leeds, Leeds, UK

5 Department of Medical Oncology, Dana-Farber Cancer Institute, Harvard Medical School, Boston, MA, USA

Department of Medicine, Memorial Sloan Kettering Cancer Center, New York, USA

7 Department of Haematology, University Hospital of Salamanca, Salamanca, Spain

8 Hopital La Mileterie, part of the Academic Hospital of Poitiers (CHU), Poitiers, France 


\begin{tabular}{|c|c|c|c|c|c|c|c|c|c|c|c|c|}
\hline Study & $\begin{array}{l}\text { Phase } \\
\text { of study }\end{array}$ & Novel agent & RCT & $\begin{array}{l}\mathrm{RR} / \geq \mathrm{CR} / \geq \mathrm{VGPR} \\
\text { in intervention arm }\end{array}$ & $\begin{array}{l}\text { Median PFS } \\
\text { (months) HR }\end{array}$ & $\begin{array}{l}\text { Median OS } \\
\text { (months) }\end{array}$ & $\begin{array}{l}\text { Median number } \\
\text { of prior lines }\end{array}$ & ASCT & $\begin{array}{l}\text { Refractory to } \\
\text { last regimen }\end{array}$ & $\begin{array}{l}\text { Bort- \& Len- } \\
\text { Refractory }\end{array}$ & Remark & $\begin{array}{l}\text { Year of } \\
\text { publication }\end{array}$ \\
\hline SIRIUS & 2 & daratumumab & No & $29.2 / 2.8 / 12.2 \%$ & $3.7 \mathrm{~m}$ & $\begin{array}{l}17.5 \mathrm{~m} 64 \% \\
\text { at } 12 \mathrm{~m}\end{array}$ & 5 & $80 \%$ & $97 \%$ & $95 \%$ & Refractory MM & $2016[21]$ \\
\hline GEN501 & 2 & daratumumab & No & $36 / 5 / 10 \%$ & $5.6 \mathrm{~m}$ & $\begin{array}{l}77 \% \text { at } 12 \mathrm{~m} \\
(16 \mathrm{mg} / \mathrm{kg})\end{array}$ & 4 & $76 \%$ & $80 \%$ & $64 \%$ & Refractory MM & 2016 [22] \\
\hline MM-003 & 3 & pomalidomide & $\begin{array}{l}\text { POM-dex } \\
\text { vs HD-Dex }\end{array}$ & $31 / 1 / 5 \%$ & $\begin{array}{l}3.8 \mathrm{~m} \text { vs } 1.9 \mathrm{~m} \\
\text { HR: } 0.48\end{array}$ & $\begin{array}{l}11.9 \text { vs } 7.8 \mathrm{~m} \\
\text { HR: } 0.74\end{array}$ & $95 \% \geq 3$ & $70 \%$ & $82 \%$ & $75 / 74 \%$ & Refractory MM & 2013 [17] \\
\hline CASTOR & 3 & daratumumab & DVd vs Vd & $83 / 19 / 59 \%$ & $\begin{array}{l}\text { NR vs } 7.2 \mathrm{~m} \\
\text { HR: } 0.39\end{array}$ & $\begin{array}{l}\text { NR in both } \\
\text { arms }\end{array}$ & 2 & $62 \%$ & $30 \%$ & Nil & $\begin{array}{l}\text { PI-refractory } \\
\text { excluded }\end{array}$ & $2016[18]$ \\
\hline POLLUX & 3 & daratumumab & DRd vs Rd & $93 / 43.1 / 75.8 \%$ & $\begin{array}{l}\text { NR vs } 18.4 \mathrm{~m} \\
\text { HR: } 0.37\end{array}$ & $\begin{array}{l}\text { NR in both } \\
\text { arms }\end{array}$ & 1 & $63 \%$ & $28 / 26.9 \%$ & Nil & $\begin{array}{l}\text { Len- refractory } \\
\text { excluded } \\
\text { MRD-ve: } 22.4 \% \\
\text { vs } 4.6 \%\end{array}$ & 2016 [23] \\
\hline Eloquent-2 & 3 & elotuzumab & $\begin{array}{l}\text { Elo-Rd vs } \\
\text { Rd }\end{array}$ & $79 / 7 / 33 \%$ & $\begin{array}{l}19.4 \mathrm{~m} \text { vs } \\
14.9 \mathrm{~m} \\
\text { HR: } 0.70\end{array}$ & $\begin{array}{l}43.7 \mathrm{~m} \text { vs } \\
39.6 \mathrm{~m} \\
\text { HR: } 0.77\end{array}$ & 2 & $55 \%$ & $35 \%$ & Nil & $\begin{array}{l}21 \% \text { bort- } \\
\text { refractory }\end{array}$ & $\begin{array}{l}2015[20, \\
45]\end{array}$ \\
\hline ASPIRE & 3 & carfilzomib & KRd vs Rd & $87 / 17.7 / 70 \%$ & $\begin{array}{l}26.3 \mathrm{~m} \text { vs } \\
17.6 \mathrm{~m} \\
\text { HR: } 0.69\end{array}$ & $\begin{array}{l}\text { NR in both } \\
\text { HR: } 0.79\end{array}$ & 2 & NR & ?Nil & Nil & & 2015 [15] \\
\hline $\begin{array}{l}\text { TOURMALINE- } \\
\text { MM1 }\end{array}$ & 3 & ixazomib & IRd vs Rd & $78 / 12 / 36 \%$ & $\begin{array}{l}20.6 \mathrm{~m} \text { vs } \\
14.7 \mathrm{~m} \\
\text { HR: } 0.74\end{array}$ & $\begin{array}{l}\text { NR in both } \\
\text { arms }\end{array}$ & $\begin{array}{l}90 \% \text { had } \leq 2 \\
\text { lines }\end{array}$ & $57 \%$ & $11 \%$ & Nil & $\begin{array}{l}\text { Bort- or Len- } \\
\text { refractory } \\
\text { excluded }\end{array}$ & $2016[16]$ \\
\hline Endeavor & 3 & carfilzomib & Kd vs Vd & $77 / 11 / 42 \%$ & $\begin{array}{l}18.7 \mathrm{~m} \text { vs } 9.4 \\
\mathrm{~m} \\
\text { HR: } 0.53\end{array}$ & $\begin{array}{l}47.6 \mathrm{~m} \text { vs } 40 \\
\mathrm{~m} \\
\text { HR: } 0.79\end{array}$ & 2 & NR & Nil & Nil & $\begin{array}{l}\text { Bort-refractory } \\
\text { excluded }\end{array}$ & $\begin{array}{l}2016 \\
{[19,26]}\end{array}$ \\
\hline
\end{tabular}

POM pomalidomide, $H D$ high-dose, $M M$ multiple myeloma, $N R$ not reached, HR hazard ratio, Bort bortezomib, Len lenalidomide, DRd daratumumab/lenalidomide/dexamethasone, KRd carfilzomib/lenalidomide/dexamethasone, IRd ixazomib/lenalidomide/dexamethasone, $K d$ carfilzomib/dexamethasone, $V d$ bortezomib/dexamethasone, $M R D$-ve minimal residual disease negativity

Correction to: Leukemia 32, 252-262 (2018); https://doi.org/10.1038/leu.2017.329; published online February 2018

Following the publication of this article the authors noted that the MRD data under the Table 1 column "Remark" of Aspire should go to that of Pollux. The authors wish to apologize for any inconvenience caused. The corrected table is attached to this correction.

Table. Major clinical trials of next generation PIs (carfilzomib, Ixazomib), next generation IMiDs (pomalidomide) and monoclonal antibody.

\section{Compliance with ethical standards}

Conflict of interest CSC received sponsorship and honoraria from Celgene, Amgen, Takeda, and Janssen. SKK received research funding for clinical trials from Celgene, Takeda, Janssen, BMS, Sanofi, Kite, Merck, Abbvie, Medimmune, Novartis, Roche-Genentech, Amgen; served as consultants or advisory board members of Celgene, Takeda, Janssen, KITE, Merck, Abbvie, Medimmune, Genentech, Amgen; and received personal honoraria from Oncopeptides, Adaptive. RZO served as advisory board member for Amgen, Bristol-Myers Squibb, Celgene, Janssen Kite Pharma, Sanofi-Aventis, and Takeda. Received research support from Amgen, BioTheryX, and Spectrum Pharma. GC received research support and honoraria from Celgene, Janssen-Cilag, and Takeda; and honoraria from Amgen, Bristol-Myers Squibb, GlycoMimetics, Seattle Genetics, Karyopharm, and Sanofi. PGR has served as a member of advisory committees to Janssen, Amgen, Celgene, Takeda, Karyopharm, and Oncopeptides, and has received research funding from Takeda, Oncopeptides, Celgene \&
Bristol Myers Squibb. MAG received personal fees from Ionis/Akcea, Alnylam, Prothena, Celgene, Janssen, Annexon, Appellis, Amgen, Medscape, Physicians Education Resource, Abbvie's Data Safety Monitoring board, Research to Practice; receives grants and personal fees from Spectrum; received speaker fees from Teva, Johnson, and Johnson, Medscape, DAVA oncology; received royalties from Springer Publishing; received grant funding from Amyloidosis Foundation \& International Waldenstrom Foundation; and served as advisory board for Pharmacyclics and Proclara outside the submitted work. SG served as advisory board members of Amgen, Actinuum, Celgene, Johnson \& Johnson, Jazz Pharmaceutical, Takeda, Novartis, Kite \& Spectrum Pharma; received research funding from Amgen, Actinuum, Celgene, Johnson \& Johnson, Miltenyi \& Takeda. MVM received honoraria from Janssen, Celgene, Amgen, Takeda, GSK, Abbvie, Seattle Genetics, Pharmamar for lectures and advisory boards. XL received honoraria from Celgene, Amgen, Takeda, BMS, Merck, Janssen, Karyopharm, Novartis, Gilead, Sanofi, Roche, Oncopeptides. KCA served as advisor/consultant to Celgene, Millennium-Takeda, Gilead, Janssen \& Bristol Myers Squibb and served as scientific founder of Oncopep \& C4 Therapeutics.

Open Access This article is licensed under a Creative Commons Attribution 4.0 International License, which permits use, sharing, adaptation, distribution and reproduction in any medium or format, as long as you give appropriate credit to the original author(s) and the source, provide a link to the Creative Commons license, and indicate if changes were made. The images or other third party material in this article are included in the article's Creative Commons license, unless indicated otherwise in a credit line to the material. If material is not included in the article's Creative Commons license and your intended use is not permitted by statutory regulation or exceeds the permitted use, you will need to obtain permission directly from the copyright holder. To view a copy of this license, visit http://creativecommons. org/licenses/by/4.0/. 